\title{
Digestive symptoms of autonomic dysfunction in Parkinson's disease patients
}

\begin{tabular}{c} 
Irene Rasanu \\
Clinical CF Hospital Constanta, Romania \\
\hline
\end{tabular}

\begin{abstract}
It is nowadays recognized that Parkinson's disease $(P D)$ involves a variety of non-motor manifestations, which sometimes seriously affect the quality of life in these patients.

This study explored the prevalence of digestive symptoms in a group of 86 PD patients from the Southeastern Romania (56\% male, mean age 70.6) who had been diagnosed with Parkinson's disease according to the United Kingdom Brain Bank criteria; patient-reported digestive symptoms were assessed using the Scale for Outcomes in Parkinson's Disease for Autonomic Symptoms (SCOPA-AUT).

The overall prevalence of digestive symptoms in the studied group was impressive $(98,8 \%)$. Defecatory dysfunction (75.6\%), ranks first followed by swallowing difficulties / choking (68.6\%) and constipation $(66.3 \%) ; 60.4 \%$ of study subjects experienced digestive symptoms "regularly" or even "often". The proportion of patients having more than 3 digestive symptoms increased almost progressively with disease duration; nevertheless, there was no statistically significant correlation between disease duration and the number or frequency of digestive symptoms.
\end{abstract}

Keywords: digestive symptoms, autonomic dysfunction, Parkinson's disease

\section{INTRODUCTION}

According to recent studies, non-motor symptoms, especially gastrointestinal dysfunctions, could be considered as early biomarkers of PD (1).

Braak postulated that PD originates in the intestine and then spreads to the brain via the vagus nerve, a phenomenon that would involve other neuronal types than dopaminergic population (1).

Autonomic dysfunction is also a common complication of Parkinson's disease $(88.46 \%$ of the patients) (2). Symptoms of autonomic nervous system (ANS) dysfunction in PD (cardiovascular, thermoregulatory, gastrointestinal, urinary, sexual) are reported among the most debilitating (3). Gastrointestinal symptoms in PD include dysphagia, nausea, constipation, and defecatory dysfunction. Unfortunately, these non motor symptoms (NMS), are very often overlooked and are poorly investigated and treated, finally resulting in a major negative impact on the clinical care and quality of life of PD patients $(3,4,5,6)$. Patients also often indicate that their NMS are more difficult to manage than their motor problems and may sometimes result in their hospitalization and institutionalization $(3,6,7)$.

When recognized, autonomic symptoms can be treated (partially, at least) resulting in improved life quality of PD patients.

The aim of this study was to assess the prevalence of patient-reported digestive symptoms in Parkinson's disease patients from the Southeastern Romania.

\section{MATERIAL AND METHODS}

Our clinical, cross-sectional, observational study was performed on 86 consecutive patients with idiopathic Parkinson's disease from 5 Outpatients Clinics of Constanta, between 01 January 2017 and 31 May 2018.

Inclusion criteria: diagnosis of idiopathic Parkinson's disease according to the UK Parkinson's Disease Society Brain Bank Diagnostic Criteria (8). 
Exclusion criteria: atypical neurological features, suggestive for other causes for parkinsonism (e.g. multiple system atrophy) were excluded.

Sex, age, disease duration (period, in years, between the diagnosis date and the date of assessment), associated medical conditions and current medications were recorded.

In order to reveal the presence of digestive symptoms, all the patients have been assessed using the Scale for Outcomes in Parkinson's Disease for Autonomic Symptoms (SCOPA-AUT) (9) as a self-administered questionnaire.

Statistical analysis included mean values, median values and/or percentages, together with the corresponding standard deviations, $95 \%$ confidence intervals and parametric tests for statistical relevance, using Word Excel and MedCalc applications.

\section{RESULTS}

Sex distribution of the studied population: 38 females (44\%) and 48 males (56\%).

The patients were aged 51 to 89 years; mean age $=70.63$ years $[\mathrm{SD}=9.74]$; mean age in women $=71.89$ years $[\mathrm{SD} 10.2]$; mean age in men $=69.64$ years [SD 9.35]. Median $=71$ years.

Disease duration varied between 1 and 16 years in the studied group; mean 6.33 years [SD 2.98]. Mean disease duration in women was 6.25 years $[\mathrm{SD}=2.87]$; mean disease duration in men was 6.35 years $[\mathrm{SD}=3.03]$.

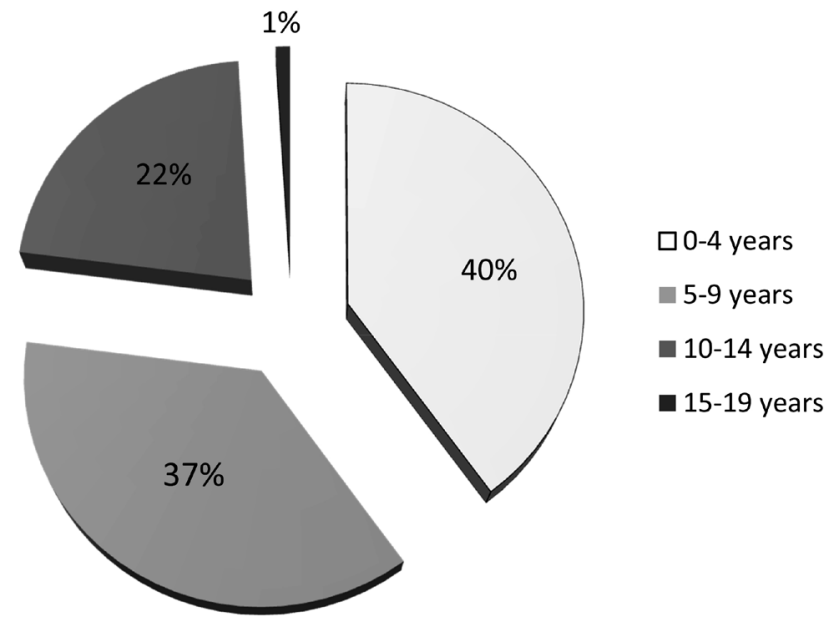

FIGURE 2. Disease duration

The overall prevalence of digestive symptoms in the studied group was impressive: $98,8 \%$ (95\% CI 96.5-100), including:

- swallowing difficulties / choke $(68.6 \%, 95 \%$ CI 58.8-78.4)

- food stuck in the throat $(62.8 \%, 95 \%$ CI CI 52.6-73)

- sialorrhea (61.6\%, 95\% CI 51.3-71.9)

- early satiety $(62.8 \%, 95 \%$ CI 52.6-73)

- constipation (defined as a bowel movement twice a week or less $)(66.3 \%, 95 \%$ CI 56.3 76.3)

- defecatory dysfunction (75.6\%, 95\% CI 66.5-84.7)

- involuntary loss of stools $(25.6 \%, 95 \% \mathrm{CI}$ 16.4-34.8)

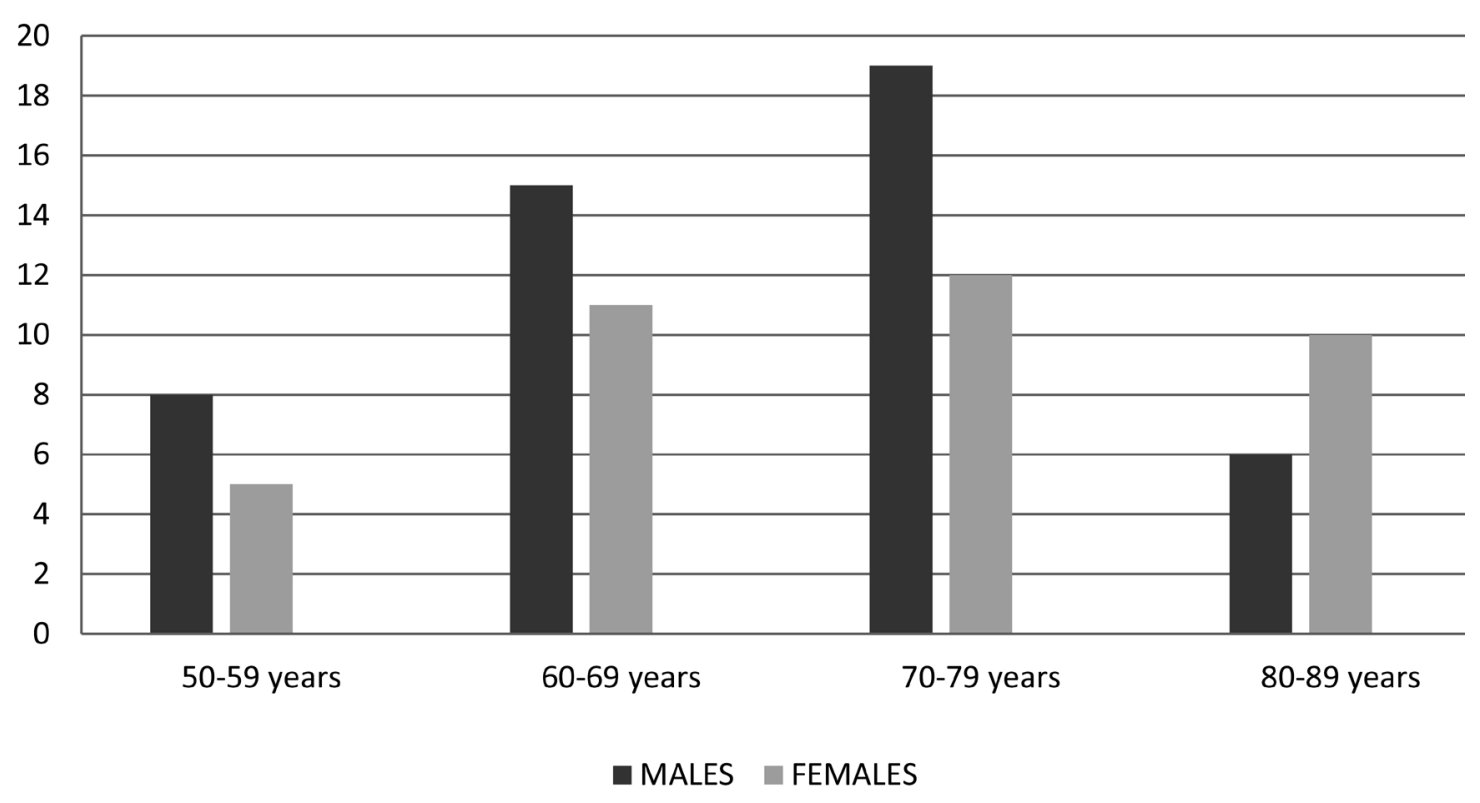

FIGURE 1. Age and sex distribution of the studied population 


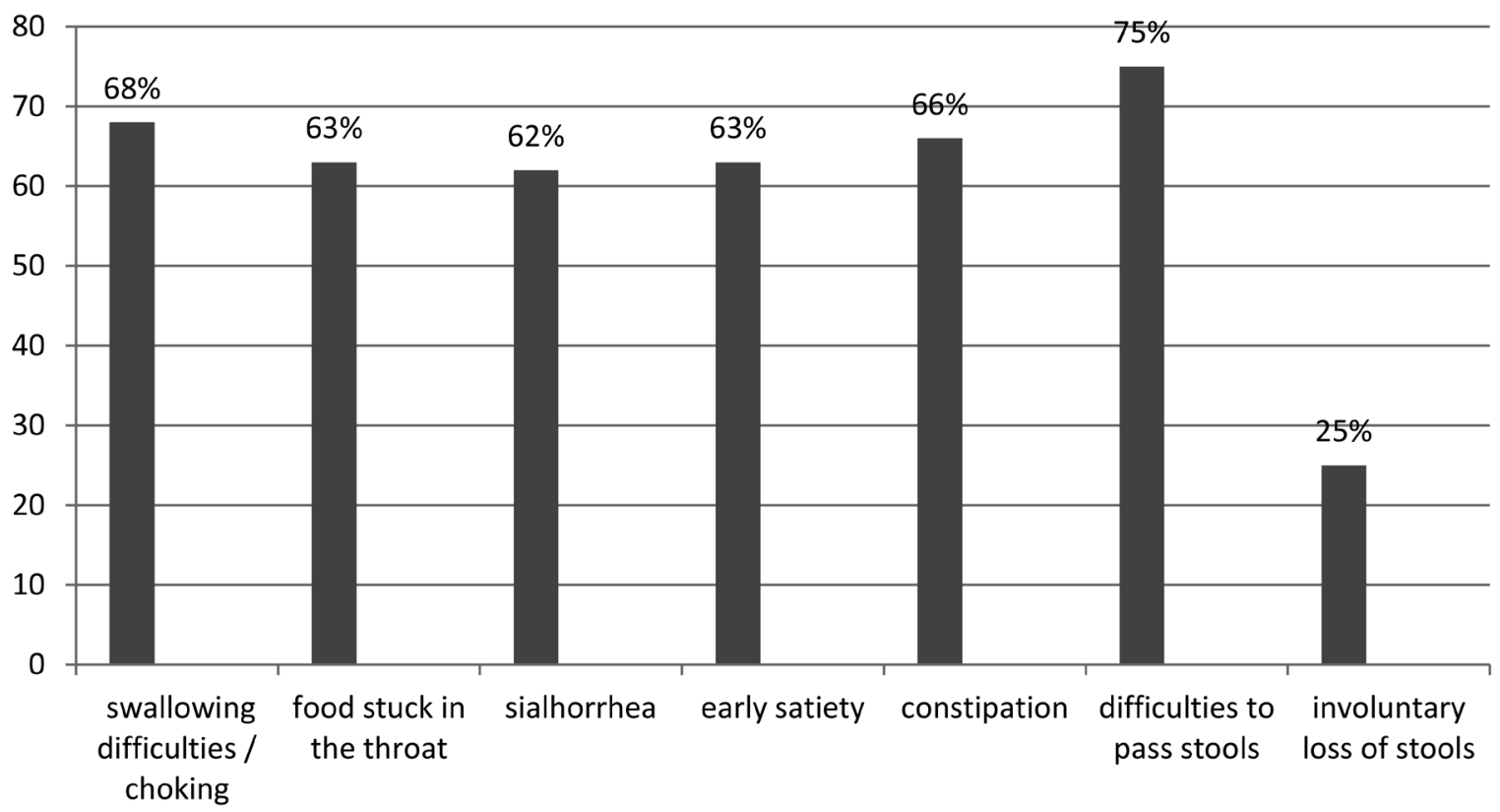

FIGURE 3. Overall prevalence of digestive symptoms

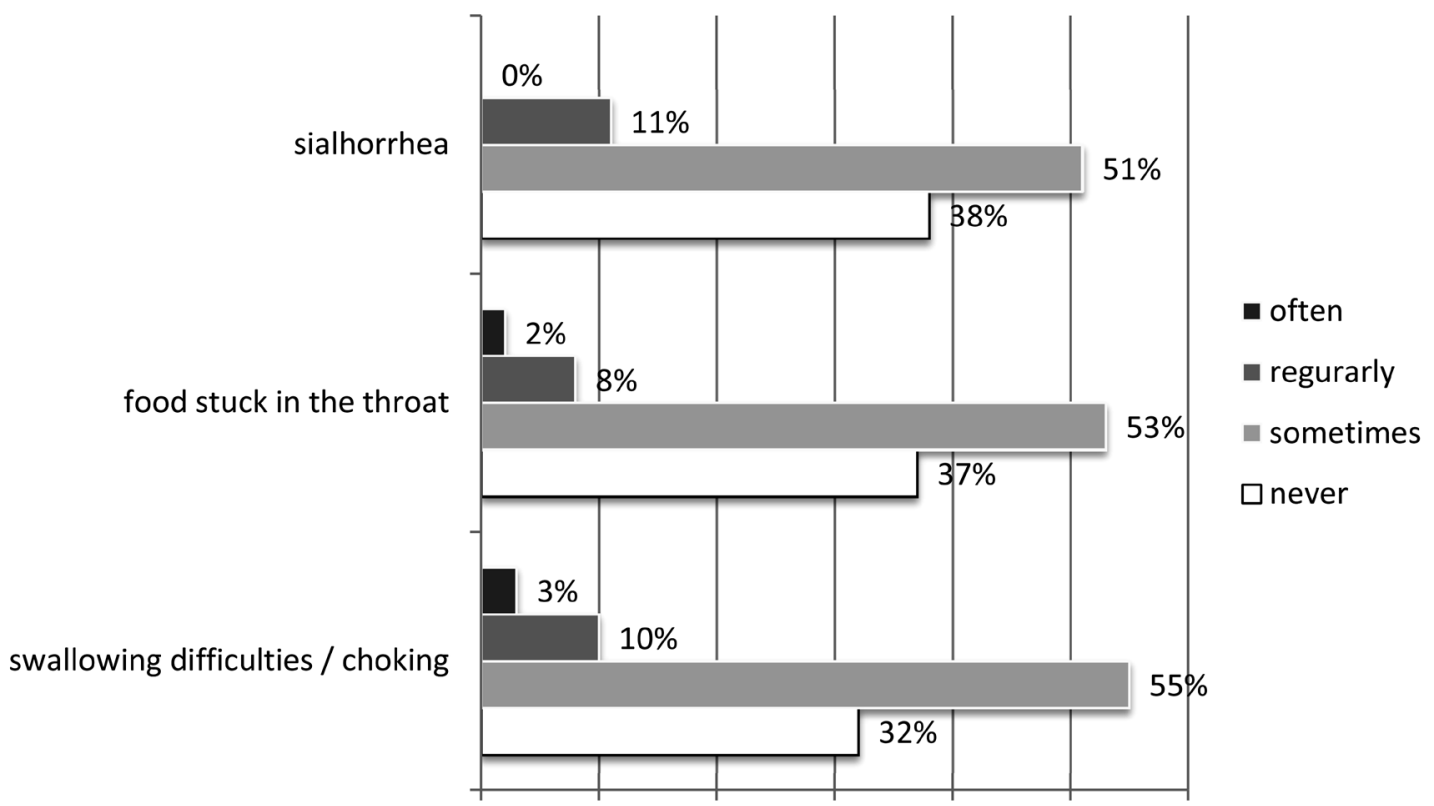

FIGURE 4. Reported frequency of deglutition and salivary symptoms

Defecatory dysfunction (75.6\%), ranks first followed by swallowing difficulties / choking $(68.6 \%)$ and constipation $(66.3 \%)$. Only $25.6 \%$ of study subject reported fecal incontinence.

As frequency, in most of the affected patients, deglutition and salivary dysfunctions as well as gastrointestinal motility dysfunctions seemed to produce symptoms "sometimes" or "regularly", but as much as $60.4 \%$ (95\% CI 50-70.7) of study subjects experienced digestive symptoms "regularly" or even "often" (Fig. 4 and 5).
In the presented study, from the 43 patients "regularly" experiencing digestive symptoms, 39.5\% (95\%CI 24.9-54.1) had less than 5 years from the PD diagnosis, $34.9 \%$ (95\%CI 20.6-49.1) between 5 and 9 years and only $25.8 \%$ (95\%CI $12.7-38.9)$ more than 10 years.

Most of the 9 patients "often" experiencing digestive symptoms $(44.4 \% 95 \% \mathrm{CI} 11.9-76.8)$ had between 5 and 9 years from the PD diagnosis and $33.3 \%$ (95\%CI 2.5-64) more than 10 years.

Among the subjects with less than 5 years from the PD diagnosis, $76.5 \%$ (95\% CI 62.2-90.7) report- 


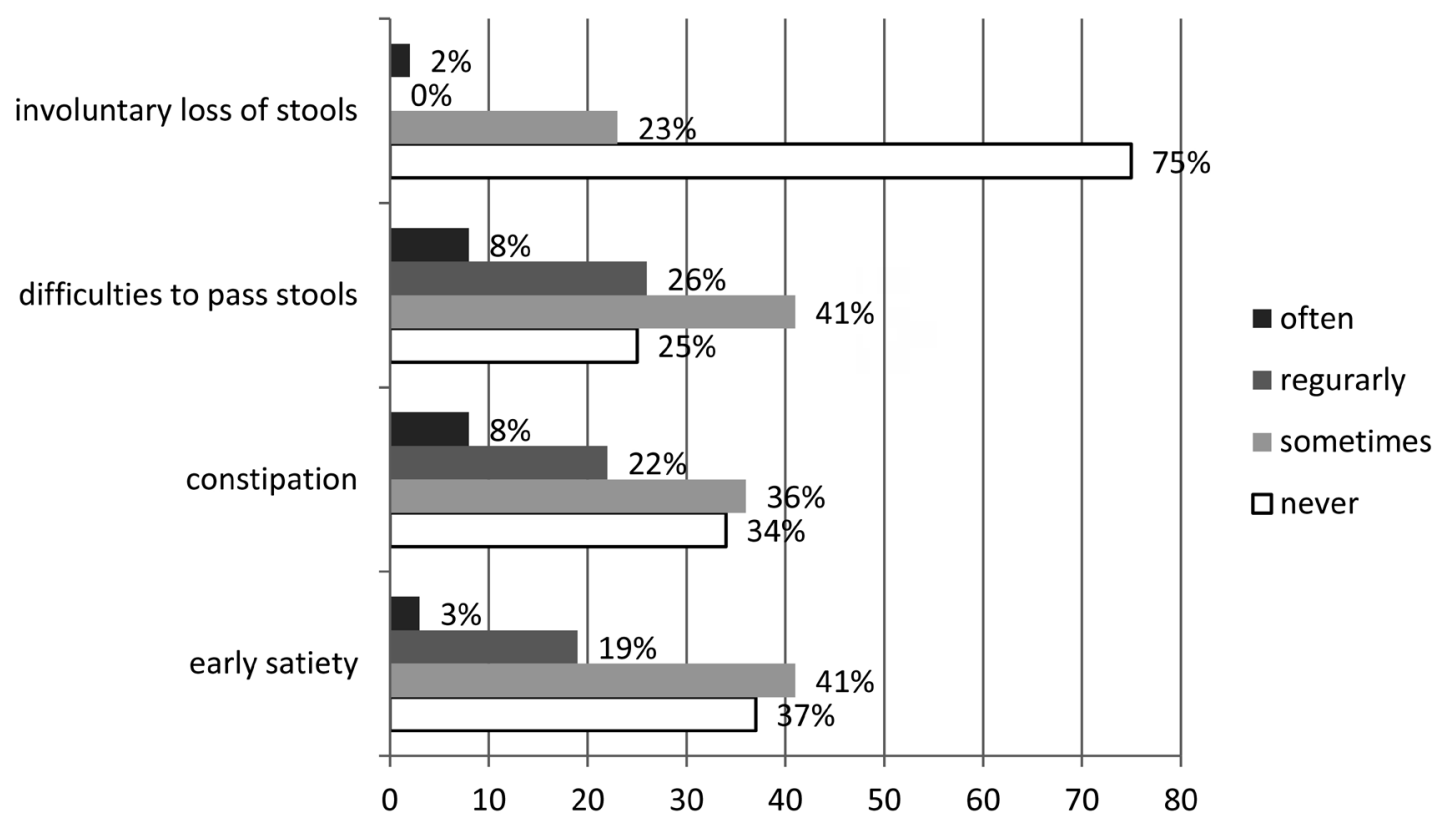

FIGURE 5. Reported frequency of gastrointestinal motility symptoms

ed more than 3 digestive symptoms, while $44.1 \%$ (95\%CI 27.4-60.8) even more than 5; the proportion of patients having more than 3 digestive symptoms increases almost progressively with disease duration.

Nevertheless, disease duration have not statistically significant correlated with the number or the frequency of digestive symptoms.

Regarding the therapy of gastrointestinal autonomic symptoms, only $45.3 \%$ of the enrolled af- fected PD patients used specific medication, and only for constipation.

\section{DISCUSSIONS}

Several studies relying on non-motor rating scales have shown that gastrointestinal manifestations occur in $60 \%$ to $80 \%$ of patients $(1,14,15,16,17)$. Untreated PD is often accompanied by autonomic nervous system impairments

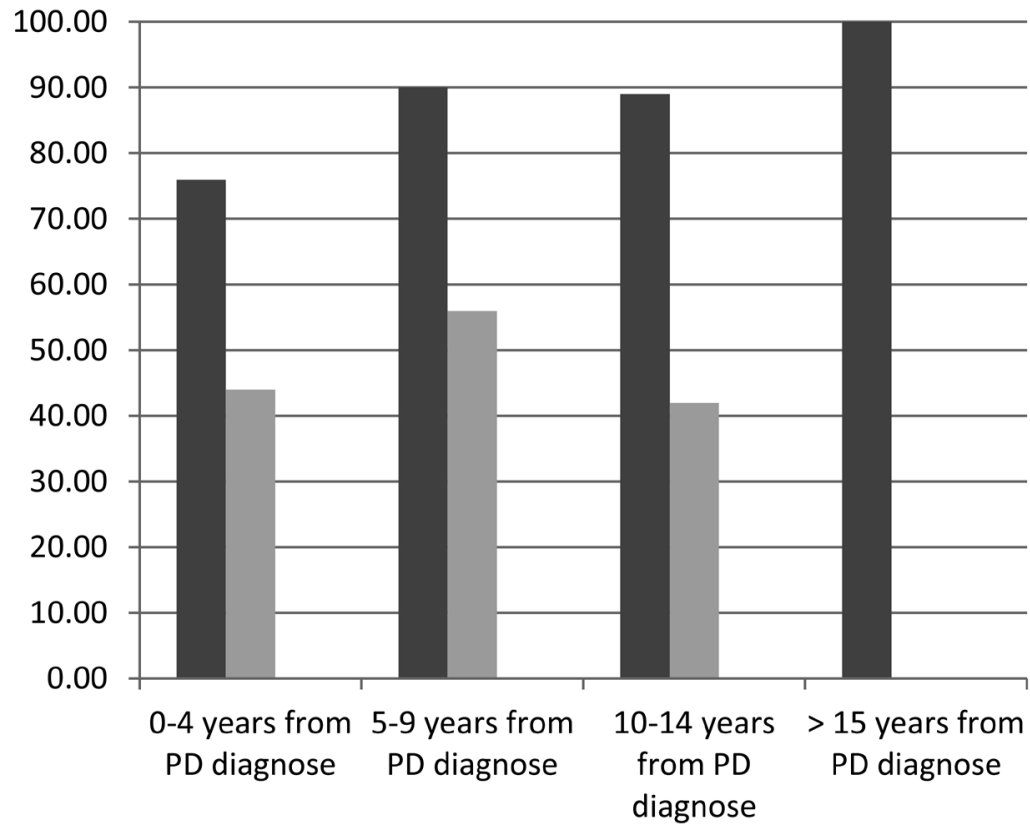

more than 3 digestive symptoms

more than 5 digestive symptoms

FIGURE 6. Number of digestive symptoms reported according to disease duration 
among which gastro-intestinal (GI) symptoms represent the most common NMS (13).

It is said that dysphagia in patients with PD is usually mild and occurs in late stages of the disease; according to international published data, it is a complaint of at least $35 \%$ of patients with PD $(3,10)$.

Pathology studies showed that in patients with $\mathrm{PD}, \alpha$-synuclein aggregates in the glossopharyngeal nerve and the pharyngeal sensory branch of the vagus nerve correlate with the severity of dysphagia $(3,11)$. Involvement of pharyngeal sensory nerves causes decreased pharyngeal sensation, contributing to dysphagia and aspiration.

Dopamine modulates salivary secretion and sialorrhea is frequently found (50 to $80 \%$ ) (3) in patients with idiopathic Parkinson's Disease (PD). Nevertheless, Bagheri et al (12) found significantly lower salivary flow in treated patients with PD and de novo patients compared to controls (probably explained also by autonomic dysfunction in PD), suggesting that in fact, reduced efficiency and frequency of swallowing result in excessive saliva.

Upper gastrointestinal symptoms reported in PD patients include: nausea, early satiety, gastric retention, and abdominal distension, caused by incomplete relaxation of esophageal sphincters, diffuse spasms, and reduced peristalsis, gastroparesis and delayed gastric emptying $(18,19,20)$.

Colonic motility is also reduced because of abnormal intrinsic (enteric nervous system - ENS which normally promotes the peristaltic reflex and extrinsic (vagal) innervations, and also because of early enteric sympathetic denervation $(21,22)$. Enteric neurons produce a substantial amount of dopamine which regulates normal intestinal motility (23). Constipation appears to be the most common autonomic and gastrointestinal symptom, reported by up to $90 \%$ of PD patients all over the world $(24,25,26)$.

Some digestive symptoms can be aggravated by the administration of L-dopa (by acting on dopaminergic enteric receptors (3).

In Romania, few published dedicated studies reported comparable data.

In 2012, the most common detected non-motor PD symptoms, among 32 patients with incipient Parkinson's disease from the northern region of Romania (detected using the revised questionnaire
NMS - PD QUEST) were the consequences of autonomic disturbances: orthostatic hypotension (81\%), nocturia (75\%), constipation (65\%) (28). Regarding other digestive symptoms, the same study reported prevalences of 53\% for defecatory dysfunction, $43 \%$ for dysphagia, $31 \%$ for sialorrhea, $21 \%$ for nausea and vomiting and only $9 \%$ for fecal incontinence.

A similar study, performed between 2007 and 2011 on 200 PD patients (in different Hoehn and Yahr stages of the disease) from the central region of Romania, revealed a prevalence of only $32 \%$ for salivary dysfunction, $30 \%$ for constipation and $15 \%$ for dysphagia (29).

Compared to these previously published studies from other regions of Romania, our study found a superior prevalence of salivary dysfunction $(61.6 \%$ versus $32 \%, \mathrm{p}<0.0001)$, dysphagia $(68.6 \%$ versus $15 \%, \mathrm{p}<0.0001)$, defecatory dysfunction $(75.6 \%$ versus $53 \%, \mathrm{p}=0.018$ ) and fecal incontinence ( $25.6 \%$ versus $9 \%, p=0.05)$, while constipation has been reported by almost the same proportion of investigated subjects $(66.3 \%$ versus $65 \%)$.

On the other hand, our study data showed that only $45.3 \%$ of the PD patients affected by gastrointestinal autonomic symptoms used specific medication, and only for constipation. This finding supports the previously reported poor recognition and treatment of non-motor symptoms in PD patients $(3,5,6)$. Left untreated, parkinsonian gastrointestinal dysfunction can lead to severe complications such as malnutrition, pulmonary aspiration, colonic volvulus, overflow diarrhea, intestinal obstruction and even intestinal perforation $(3,14,15,16,17)$; that's why GI disorders have been reported among the most common causes of emergency admission in PD patients (13).

In addition, it has been demonstrated that treating non-motor symptoms improves the quality of life of PD patients, particularly those who positively respond to a dopaminergic therapy (2).

On our knowledge, this is the first published study concerning the prevalence of digestive symptoms in parkinsonian patients performed in Southeastern Romania. The continuation of the study, including more PD patients and more clinical parameters would increase the statistical significance of the results. 


\section{CONCLUSIONS}

The overall prevalence of digestive symptoms in the studied group was impressive $(98,8 \%)$. Defecatory dysfunction, ranks first followed by swallowing difficulties / choking and constipation. The majority of study subjects experienced digestive symptoms "regularly" or even "often".

The proportion of patients having more than three digestive symptoms increased almost progressively with disease duration. Nevertheless, there was no statistically significant correlation between disease duration and the number or frequency of digestive symptoms.

\section{REFERENCES}

1. Poirier AA, Aubé B, Côté M, Morin N, Di Paolo T, Soulet D. Gastrointestinal Dysfunctions in Parkinson's Disease: Symptoms and Treatments. Parkinsons Dis. 2016; 2016:6762528.

2. Karbozova K. Assessment of Autonomic Dysfunction in Parkinson's Disease. Abstr. In. Mov Disord. 2017; 32 (suppl 2).

3. Palma JA, Kaufmann H. Treatment of Autonomic Dysfunction in Parkinson Disease and Other Synucleinopathies. Movement Disorders. 2018; Vol. 33, No. 3, 372-390.

4. Sauerbier A, Chaudhuri KR. Non-motor symptoms: the core of multi-morbid Parkinson's disease. British Journal of Hospital Medicine. 2014; vol. 75, no. 1, 18-24.

5. Soh SE,Morris ME, McGinley JL. Determinants of health-related quality of life in Parkinson's disease: a systematic review. Parkinsonism and Related Disorders. 2011; vol. 17, no. 1, 1-9.

6. Todorova A, Jenner P, Chaudhuri KJ. Non-motor parkinson's: integral to motor parkinson's, yet often neglected. Practical Neurology. 2014; vol. 14 , no. 5, 310-322.

7. Bernal-Pacheco O, Limotai N, Go CL, Fernandez HH. Nonmotor manifestations in parkinson disease. Neurologist. 2012; vol. 18, no. 1, 1-16.

8. Hughes AJ, Daniel SE, Kilford L, Lees AJ. Accuracy of clinical diagnosis of idiopathic Parkinson's disease: a clinico-pathological study of 100 cases. Journal of Neurology, Neurosurgery, and Psychiatry 1992;55:181-184

9. Visser M, Marinus J, Stiggelbout AM, Van Hilten JJ. Assessment of autonomic dysfunction in Parkinson's disease: the SCOPA-AUT. Mov Disord 2004; 19:1306-12

10. Seppi K, Weintraub D, Coelho M et al. The movement disorder society evidence-based medicine review update: treatments for the non-motor symptoms of Parkinson's disease. Movement Disorders. 2011; vol. 26, supplement 3, S42-S80.

11. Strauss I, Kalia SK, Lozano AM. Where are we with surgical therapies for Parkinson's disease? Parkinsonism and Related Disorders. 2014; vol. 20, no. 1, S187-S191.

12. Bagheri H, Damase-Michel C, Lapeyre-Mestre M et al. A study of salivary secretion in Parkinson's disease. Clin Neuropharmacol. 1999 Jul-Aug;22(4):213-5.

13. Cloud LJ, JG Greene JG. Gastrointestinal features of Parkinson's disease. Current Neurology and Neuroscience Reports 2011; vol. 11, no. 4, 379-384.

14. Lyons KE, Pahwa R. The impact and management of nonmotor symptoms of Parkinson's disease. American Journal of Managed Care. 2011; S308-S314.
Less than a half of the investigated PD patients affected by gastrointestinal autonomic symptoms used specific medication, and only for constipation, This alarming finding supports the previously reported poor recognition and treatment of non-motor symptoms in PD patients

\section{Acknowledgements}

I would like to thank Raican George and Darawsha Khalil for their help to accomplish this work.

Conflict of interest: none declared Financial support: none declared
15. Guneysel O, Onultan O, Onur O. Parkinson's disease and the frequent reasons for emergency admission. Neuropsychiatric Disease and Treatment. 2008; vol. 4, no. 4, 711-714.

16. Dubow JS. Autonomic dysfunction in Parkinson's disease. Diseasea-Month. 2007; vol. 53, no. 5, 265-274.

17. Barone $\mathrm{P}$, Antonini A, Colosimo $\mathrm{C}$ et al. The PRIAMO study: a multicenter assessment of nonmotor symptoms and their impact on quality of life in Parkinson's disease. Movement Disorders. 2009; vol. 24, no. 11, 1641-1649.

18. Thomaides T, Karapanayiotides T, Zoukos $Y$ et al. Gastric emptying after semi-solid food in multiple system atrophy and Parkinson disease. J Neurol 2005;252:1055-1059.

19. Suttrup I, Suttrup J, Suntrup-Krueger S et al. Esophageal dysfunction in different stages of Parkinson's disease. Neurogastroenterol Motil $2017 ; 29$.

20. Su A, Gandhy R, Barlow C, Triadafilopoulos G. Clinical and manometric characteristics of patients with Parkinson's disease and esophageal symptoms. Dis Esophagus 2017;30:1-6.

21. Sakakibara R, Uchiyama T, Yamanishi T et al. Bladder and bowel dysfunction in Parkinson's disease. Journal of Neural Transmission. 2008; vol. 115, no. 3, 443-460.

22. Arnhold M, Dening Y, Chopin M et al. Changes in the sympathetic innervation of the gut in rotenone treated mice as possible early biomarker for Parkinson's disease. Clin Auton Res 2016;26: 211-222.

23. Pfeiffer RF. Gastrointestinal dysfunction in Parkinson's disease. The Lancet Neurology. 2003; vol. 2, no. 2, 107-116.

24. Park H, Lee JY, Shin CM, Kim JM, Kim TJ, Kim JW. Characterization of gastrointestinal disorders in patients with parkinsonian syndromes. Parkinsonism Relat Disord 2015;21:455-460.

25. Verbaan D, Marinus J, Visser M, van Rooden SM, Stiggelbout AM, van Hilten JJ. Patient-reported autonomic symptoms in Parkinson disease. Neurology 2007;69:333-341.

26. Knudsen K, Krogh K, Ostergaard K, Borghammer P. Constipation in parkinson's disease: Subjective symptoms, objective markers, and new perspectives. Mov Disord 2017;32:94-105.

27. Arunraj Ezhumalai, Ravikumar V. Autonomic nervous system dysfunction in Parkinson's disease patients. Int J Res Med Sci. 2017 Jul;5(7):2895-2904.

28. Tohănean N, Perju-Dumbravă L. Evaluarea simptomelor nonmotorii la pacienții cu boală Parkinson precoce. Clujul Medical. 2012; Vol. 85 - nr. 2, 212-217.

29. Cuibus L, Pereanu M. Tulburări vegetative în boala Parkinson. ActaMedicaTransilvana. 2012; vol II, nr. 2, 2012, pag. 12-16. 